\title{
Identification of a New Selective Chemical Inhibitor of Mutant Isocitrate Dehydrogenase-1
}

SHORT

COMMUNICATION

\author{
Hyo-Joon Kim ${ }^{1}$, Bu Young Choi ${ }^{2}$, Young-Sam Keum ${ }^{1}$ \\ 'College of Pharmacy, Dongguk University, Goyang, ${ }^{2}$ Department of Pharmaceutical Science and Engineering, Seowon University, Cheongju, Korea
}

\begin{abstract}
Background: Recent genome-wide sequencing studies have identified unexpected genetic alterations in cancer. In particular, missense mutations in isocitrate dehydrogenase-1 (IDH1) at arginine 132, mostly substituted into histidine (IDH1-R132H) were observed to frequently occur in glioma patients.

Methods: We have purified recombinant IDH1 and IDH1-R132H proteins and monitored their catalytic activities. In parallel experiments, we have attempted to find new selective IDH1-R132H chemical inhibitor(s) from a fragment-based chemical library.

Results: We have found that IDH1, but not IDH1-R132H, can catalyze the conversion of isocitrate into $\alpha$-ketoglutarate ( $\alpha$-KG). In addition, we have observed that IDH1-R132H was more efficient than IDH1 in converting $\alpha$-KG into $(R)$-2-hydroxyglutarate $(R-2 \mathrm{HG})$. Moreover, we have identified a new hit molecule, e.g., 2-(3-trifluoromethylphenyl)isothioazol-3(2H)-one as a new selective IDH1-R132H inhibitor. Conclusions: We have observed an underlying biochemical mechanism explaining how a heterozygous IDH1 mutation contributes to the generation of $\mathrm{R}-2 \mathrm{HG}$ and increases cellular histone $\mathrm{H} 3$ trimethylation levels. We have also identified a novel selective IDH1-R132H chemical hit molecule, e.g., 2-(3-trifluoromethylphenyl)isothioazol-3(2H)-one, which could be used for a future lead development against IDH1-R132H.
\end{abstract}

(J Cancer Prev 2015;20:78-83)

Key Words: Isocitrate dehydrogenase-1, Isocitrate, $\alpha$-ketoglutarate, (R)-2-hydroxyglutarate, 2-(3-trifluoromethylphenyl)isothioazol-3(2H)-one

\section{INTRODUCTION}

Cancer is an example of common human disease, in which cellular metabolisms are significantly altered, thereby exhibiting a substantially different production of cellular metabolites. ${ }^{1}$ More than decades ago, Warburg ${ }^{2}$ has observed that proliferating cancer cells preferentially convert the majority of glucose to lactate, but not to the oxidative phosphorylation for glucosedependent adenosine triphosphate production even in oxygenrich conditions, a phenomenon commonly referred to as 'the Warburg effect'. Although no single genetic aberration is common to all cancer types, the metabolic switch into the aerobic glycolysis is universal to nearly all proliferating cancer cells, regardless of their origin. Therefore, this property has been exploited in the clinic for diagnosis of cancer, using ${ }^{18} \mathrm{~F}$-deoxyglucose positron emission tomography.

Results from recent genome-wide sequencing studies have illustrated that somatic mutations in the isocitrate dehydrogenase-1 (IDH1) commonly exist in the majority of patients with grade II to III primary gliomas and secondary glioblastomas multiforme. ${ }^{3}$ Interestingly, IDH1 mutations were confined at a single residue, e.g., arginine 132 , which was mostly mutated into histidine (IDH1-R132H). ${ }^{4} \mathrm{IDH} 1$ is a cytosolic enzyme that catalyzes the oxidative decarboxylation of isocitrate (ICT) into $\alpha$-ketoglutarate $(\alpha-\mathrm{KG})$ with a concomitant production of reduced nicotinamide adenine dinucleotide phosphate (NADPH). Structural analysis shows that IDH1 arginine residue at 132 is located in the active site of the enzyme and participates in the binding of

Received February 7, 2015, Revised March 7, 2015, Accepted March 9, 2015

Correspondence to: Young-Sam Keum

College of Pharmacy, Dongguk University, 32 Dongguk-ro, Ilsandong-gu, Goyang 410-820, Korea

Tel: +82-31-961-5215, Fax: +82-31-961-5206, E-mail: keum03@dongguk.edu

Copyright (C) 2015 Korean Society of Cancer Prevention

(c) This is an Open Access article distributed under the terms of the Creative Commons Attribution Non-Commercial License (http://creativecommons. org/icenses/by-nc/3.0) which permits unrestricted non-commercial use, distribution, and reproduction in any medium, provided the original work is properly cited. 
ICT. ${ }^{5}$ In addition, somatic IDH1 mutations were observed to be heterozygous: only a single copy of the homologous genes is mutated. ${ }^{6}$

Because the enzymes, mutated in the active site generally exhibit decreased enzymatic activities, IDH1 mutations were thought to cause a loss of function of enzyme. ${ }^{7}$ However, it was soon noticed that IDH1-R132H exhibits an additional novel enzyme activity: it converts $\alpha$-KG into a stereoselective (R)-2-hydroxyglutarate $(R-2 \mathrm{HG}){ }^{8}{ }^{8}$ Subsequent studies have demonstrated that treatment of $R-2 \mathrm{HG}$ is sufficient to promote reversible leukemogenesis in vitro ${ }^{9}$ and glioma patients harboring IDH1 mutations had an increased $R$-2HG level in the brain, ${ }^{10}$ suggesting a possibility that $R-2 \mathrm{HG}$ might be an oncometabolite. In the present study, we have measured the catalytic activity of recombinant IDH1 and IDH1-R132H enzymes and found out that a heterozygous IDH1-R132H mutation might be required to fulfill the two-step enzymatic conversion of ICT into $R$-2HG. In addition, we have identified a new selective IDH1-R132H chemical hit molecule from a fragment-based chemical library.

\section{MATERIALS AND METHODS}

\section{Preparation of recombinant IDH1 and IDH1-R132H proteins}

Human IDH1 cDNA (GenBank Number, AF020038) was purchased from Korea Human Gene Bank (Daejeon, Korea). A site-directed mutagenesis using an overlapping polymerase chain reaction was conducted to create a mutant IDH1 CDNA (IDH1R132H). Both wild-type and mutant IDH1 CDNAs were subcloned into the pET21 vector and transformed into BL21 cells. Cells were grown in $\mathrm{LB}$ media at $37^{\circ} \mathrm{C}$ until $\mathrm{OD}_{600}$ reaches at the absorbance of 0.6. Recombinant proteins were induced and prepared by adding isopropyl- $\beta$-D-thiogalacto-pyranoside (IPTG) with a final concentration of $1 \mathrm{mM}$ for 4 hours. Cell were resuspended in cell lysis buffer (20 mM Tris-Cl, pH 7.4, 0.1\% [v/v] Triton X-100, 500 $\mathrm{mM} \mathrm{NaCl}, 1 \mathrm{mM}$ phenylmethylsulfonyl fluoride [PMSF], $5 \mathrm{mM}$ $\beta$-mercaptoethanol, 10\% [v/v] glycerol) and heavily sonicated in 4 times for every 30 seconds. Samples were centrifuged at 12,000 rpm for 1 hour and supernatant was loaded in $\mathrm{Ni}^{2+}$-affinity resin (GE Healthcare, Piscataway, NJ, USA), which was previously activated with buffer 1 (20 mM Tris-Cl, pH 7.4, $500 \mathrm{mM} \mathrm{NaCl}, 5$ $\mathrm{mM} \beta$-mercaptoethanol, 10\% [v/v] glycerol). Resin was washed by buffer 1 three times and the sample elution was performed with an appropriate volume of buffer $2(20 \mathrm{mM}$ Tris-Cl, pH 7.4, $500 \mathrm{mM}$ $\mathrm{NaCl}, 5 \mathrm{mM}$ 3-mercaptoethanol, $500 \mathrm{mM}$ imidazole, 10\% [v/v] glycerol). Eluted samples were dialyzed twice with buffer 3
(50mM Tris-Cl, pH 7.4, $200 \mathrm{mM} \mathrm{NaCl}, 5 \mathrm{mM}$ 3-mercaptoethanol, $2 \mathrm{mM} \mathrm{MnSO}_{4}, 10 \%[\mathrm{v} / \mathrm{v}]$ glycerol) and stored at $-80^{\circ} \mathrm{C}$ for future biochemical analyses.

2. Measurement of IDH1 and IDH1-R132H activity and exploration of novel selective IDH1-R132H inhibitor(s) from fragment-based chemical library

The IDH activity was assayed by measuring the reduction of $\mathrm{NADP}^{+}$into NADPH or the oxidation of NADPH into NADP ${ }^{+}$with spectrophotometry, based on the principle that NADPH, but not $\mathrm{NADP}^{+}$, possesses an optical absorption at the wavelength of 340 $\mathrm{nm}$. In order to measure the reduction of $\mathrm{NADP}^{+}$into $\mathrm{NADPH}, 1$ $\mu \mathrm{g}$ recombinant protein was added to $200 \mu \mathrm{L}$ assay solution (100 $\mathrm{mM}$ Tris-Cl, $\mathrm{pH} 7.5,1.3 \mathrm{mM} \mathrm{MnCl} 2,0.33 \mathrm{mM}$ ethylenediaminetetraacetic acid (EDTA), $0.1 \mathrm{mM} \beta-\mathrm{NADP}^{+} 0.1 \mathrm{mM} \mathrm{D}(+)$-threoisocitrate) and the resulting absorbance was measured at $340 \mathrm{~nm}$ every 5 minutes. In order to measure the oxidation of $\mathrm{NADPH}$ into $\mathrm{NADP}^{+}, 1 \mu \mathrm{g}$ recombinant protein added to $200 \mu \mathrm{L}$ assay solution (100 mM Tris-Cl, pH 7.5, 1.3 mM MnCl $2,0.33$ mM EDTA, $0.1 \mathrm{mM} \beta$-NADPH, $0.5 \mathrm{mM} \alpha-\mathrm{KG}$ ) and the decreasing absorbance was also measured at $340 \mathrm{~nm}$. Exploration of novel selective chemical inhibitor(s) for IDH1- R132H was attempted with the above reaction setup with an addition of individual chemical inhibitors at the concentration of $10 \mu \mathrm{M}$. The fragment-based chemical library (Maybridge Ro3 chemical library) used in the study was purchased from Maybridge Company (Cambridge, UK).

\section{Transient transfection and Western blot analysis}

293T cells were grown in Dulbecco's Modified Eagle's Medium media supplemented with $10 \%$ fetal bovine serum. Seventy percent confluent 293T cells in $100 \mathrm{~mm}$ dish were transfected with $3 \mu \mathrm{g}$ pcDNA3- HA-IDH1 or pcDNA3-HA-IDH1-R132H plasmids with JetPEI reagent (Polyplus Transfection, New York, NY, USA). After 24 hours transfection, 293T cell lysates were collected with $200 \mu \mathrm{L}$ sodium dodecyl sulfate (SDS) lysis buffer $(50 \mathrm{mM}$ Tris-HCl at pH 8.0, $150 \mathrm{mM} \mathrm{NaCl}, 1 \% \mathrm{NP}-40,0.5 \%$ deoxycholic acid, $1 \% \mathrm{SDS}, 1 \mathrm{mM} \mathrm{Na}_{3} \mathrm{VO}_{4}, 1 \mathrm{mM}$ dithiothreitol, $1 \mathrm{mM}$ PMSF) and kept on ice for 30 minutes. After centrifugation at $12,000 \mathrm{rpm}$ for 10 minutes, the protein concentration was measured using a BCA Protein Assay Kit (Thermo Fisher Scientific, Waltham, MA, USA). An equal amount of cell lysates were resolved by SDS-polyacrylamide gel electrophoresis and transferred to polyvinylidene difluoride membranes (BioRad, Hercules, CA, USA). The membranes were incubated in blocking buffer ( $5 \%$ skim milk in 1 $\times$ phosphate buffered saline [PBS]-0.1\% Tween-20, [PBST]) for 1 hour and hybridized with the appropriate primary antibodies in 
$1 \times$ PBS, containing $3 \%$ bovine serum albumin or $3 \%$ skim milk overnight at $4^{\circ} \mathrm{C}$. Primary antibodies used in our study are as follows: H3K9me3 (abcam, Cambridge, UK; ab8898), H3K27me3 (Millipore, Billerica, MA, USA; 07-449), H3K36me3 (abcam; ab9050), H3K79me3 (abcam; ab2621), total H3 (Millipore; 05-928) and HA (Cell Signaling Technology, Danvers, MA, USA; 2367). After washing three times with $1 \times$ PBST for 30 minutes, the membrane was hybridized with horseradish peroxidase-conjugated secondary antibody (Santa Cruz Biotechnology, Santa Cruz, CA, USA) for 1 hour at room temperature and washed three times with $1 \times$ PBST solution for 30 minutes. The membrane was visualized by using an enhanced chemiluminescence detection system.

\section{RESULTS}

\section{Recombinant IDH1-R132H do not convert $\alpha-K G$ into $\mathrm{R}-2 \mathrm{HG}$}

We have prepared recombinant IDH1 and IDH1-R132H enzymes via IPTG induction in Escherichia coli, followed by a nickel-based affinity purification and dialysis. Coomassie blue staining illustrates that both IDH1 and IDH1-R132H proteins are soluble (Fig. 1A). Because IDH1 requires $\mathrm{NADP}^{+}$as a cofactor, the IDH1 activity converting ICT into $\alpha-\mathrm{KG}$ can be indirectly quantified by measuring the amount of resulting NADPH at 340 $\mathrm{nm}$ by spectrophotometry (Fig. 1B, upper panel). Our results show that IDH1 efficiently converts ICT into $\alpha$-KG through a coupled reduction of $\mathrm{NADP}^{+}$into NADPH, while IDH1-R132H fails to do so (Fig. 1B, lower panel). As stated earlier, it is known that IDH1-R132H gains a new enzyme activity, e.g., catalysis of $\alpha$-KG into $R-2 \mathrm{HG}$, which can be indirectly assessed by measuring the formation of $\mathrm{NADP}^{+}$from NADPH (Fig. 1C, upper panel). However, it is unclear whether this activity also occurs in IDH1. Our result illustrates that both IDH1 and IDH1-R132H can convert $\alpha-\mathrm{KG}$ into $\mathrm{R}-2 \mathrm{HG}$, although the degree of this metabolic conversion by IDH1-R132H was far more efficient, when compared with that by IDH1 (Fig. 1C, lower panel).

\section{Overexpression of IDH1-R132H strongly elevated the H3K36me3 and H3K79me3 levels in HEK293T cells}

Previous studies have demonstrated that $R-2 \mathrm{HG}$ acts as an antagonist of $\alpha-\mathrm{KG}$ to competitively inhibit a number of KGdependent dioxygenases, including the JmjC domain-containing histone demethylases (KDMs) and the TET (ten-eleven translocation) family of DNA hydroxylases. ${ }^{11}$ We have transfected pcDNA3 vector, pcDNA3-HA-IDH1 and pcDNA3-HA-IDH1-R132H plasmids into HEK293T cells and measured the resulting global changes of histone $\mathrm{H} 3$ trimethylation levels at Lysine 9 (H3K9me3), Lysine 27 (H3K27me), Lysine 36 (H3K36me3) and Lysine 79 (H3K79me3). As a result, we observed that overexpression of IDH1-R132H in 293T cells significantly increased the endogenous levels of H3K36me3 and H3K79me3, but not that of H3K9me3 and H3K27me3 (data not shown), when compared with 293 T cells overexpressed with wild-type IDH1 (Fig. 1D). The exact reason why $\mathrm{H} 3 \mathrm{~K} 9$ me3 and $\mathrm{H} 3 \mathrm{~K} 27$ me3 levels were unaffected by overexpression of IDH1-R132H in our experiment setup is currently unclear. Our results suggest that IDH-R132H might elevate the H3K36me3 and H3K79me3 levels in HEK293T cells, possibly via the production of $R-2 \mathrm{HG}$.

\section{Identification of a novel selective IDH1-R132H inhibitor from fragment-based chemical library}

In order to find out new selective IDH1-R132H chemical inhibitors, we have screened 500 synthetic chemicals derived from a fragment-based chemical library at the concentration of 5 $\mu \mathrm{M}$. While several synthetic chemicals exhibited mild inhibitory effects, we observed that a synthetic fragment chemical (No. 398) strongly inhibited the IDH1-R132H activity more than 80\% (Fig. 2A). The chemical was identified to be 2-(3-trifluoromethylphenyl)isothioazol-3(2H)-one and its chemical structure is provided herein (Fig. 2B). In order to exclude the possibility whether this compound also interferes with the wild-type IDH1 activity, the recombinant IDH1 protein was mixed with 2-(3-trifluoromethylphenyl)isothioazol-3(2H)-one and the resulting enzymatic activity was assessed. As a result, we observed that 2-(3-trifluoromethylphenyl)isothioazol-3(2H)-one does not interfere with the IDH1 activity, demonstrating the selectivity of this compound against IDH1-R132H (Fig. 2C).

\section{DISCUSSION}

As stated earlier, the next-generation sequencing studies have identified that the IDH1 mutations found in glioma patients exhibit a heterozygous, but not homozygous pattern. Our data enable us to speculate the biochemical mechanisms how a heterozygous IDH1-R132H mutation contributes to the formation of $R-2 \mathrm{HG}$ in vivo: if the IDH1 mutation is homozygous, the generation of $R$-2HG from ICT would be impossible because IDH1-R132H can't convert ICT into $\alpha$-KG (Fig. 1B). This might explains why heterozygous, but not homozygous IDH1 mutations are exclusively found in glioma patients. In addition, we have demonstrated that 2-(3-trifluoromethylphenyl)isothioazol-3(2H)-one 

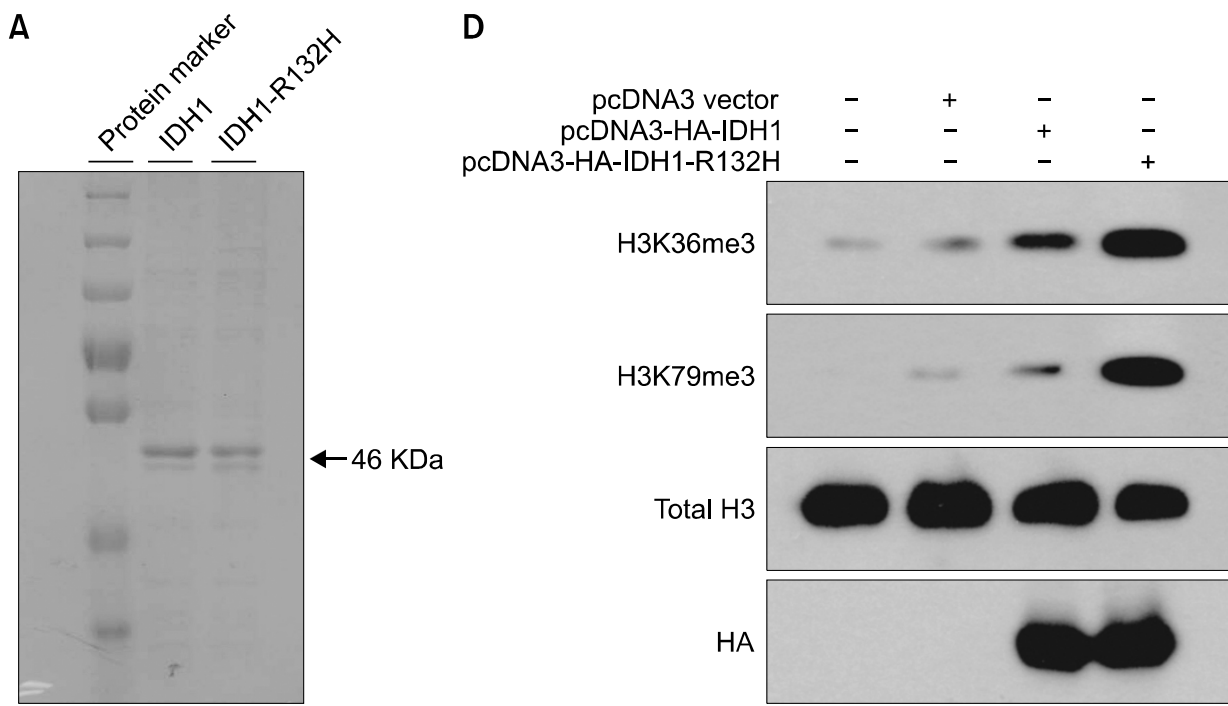

B

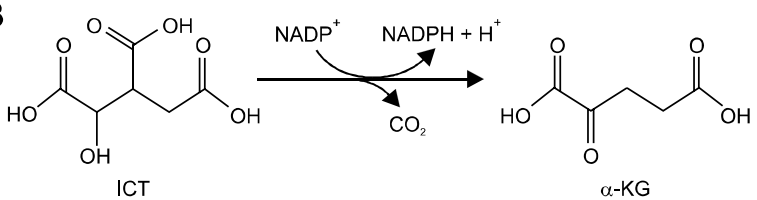

C

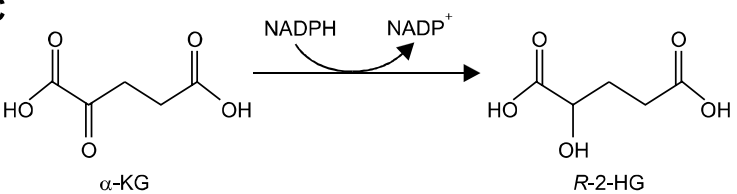
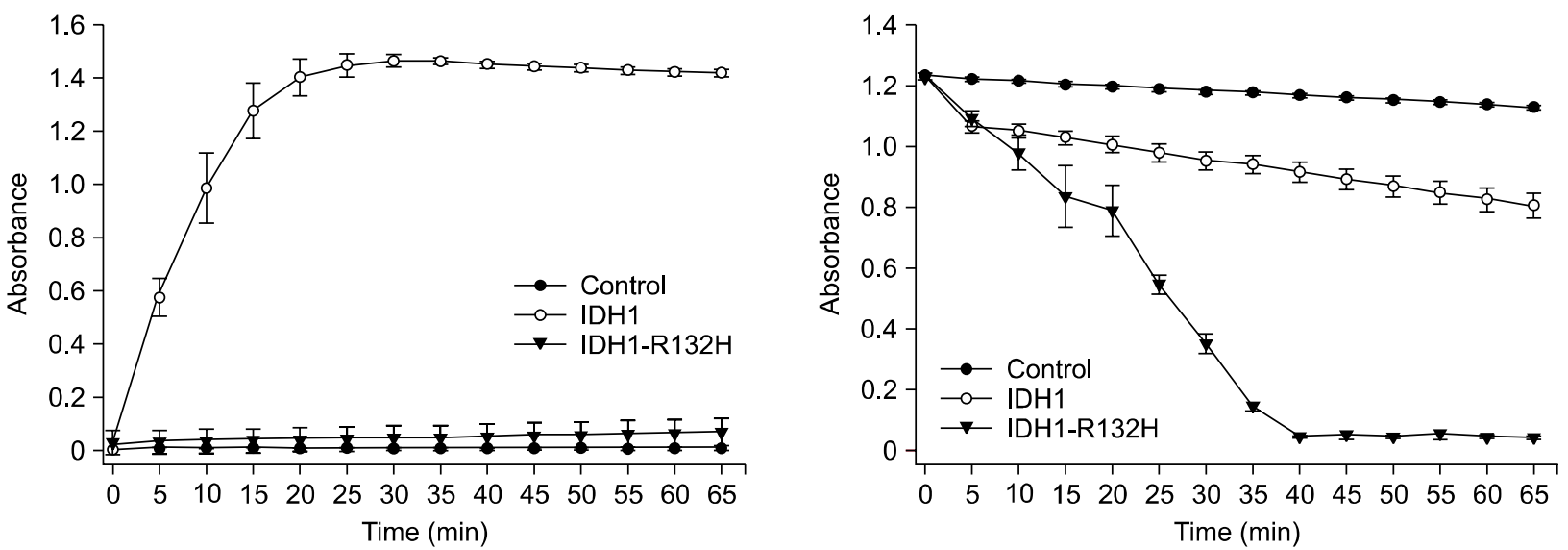

Figure 1. Measurement of the catalytic activities of recombinant isocitrate dehydrogenase-1 (IDH1) and IDH1-R132H enzymes. (A) Purification of recombinant IDH1 and IDH1-R132H proteins. (B) Measurement of IDH1 and IDH1-R132H activities converting isocitrate (ICT) into $\alpha$-ketoglutarate $(\alpha-K G)$ by measuring the reduction of $\mathrm{NADP}^{+}$into nicotinamide adenine dinucleotide phosphate (NADPH). The enzyme activity is plotted as an increasing absorbance at the $320 \mathrm{~nm}$ wavelength. (C) Measurement of IDH1 and IDH1-R132H activities converting $\alpha$-KG into a stereoselective $(R)$-2-hydroxyglutarate $(R-2 \mathrm{HG})$ by measuring the oxidation of NADPH into $\mathrm{NADP}^{+}$. The enzyme activity is plotted as a decreasing absorbance at the $320 \mathrm{~nm}$ wavelength. (D) The effect of IDH1 and IDH1-R132H on cellular histone H3 trimethylation levels. The 293T cells was transfected with pcDNA3 vector, pcDNA3-HA-IDH1 and pcDNA3-HA-IDH1-R132H plasmids and Western blot was conducted with indicated primary antibodies.

inhibits the IDH1-R132H activity without affecting the IDH1 activity (Fig. 2A and 2C). In line with our discovery, other investigators have reported selective chemical inhibitors of IDH1-R132H. For example, Rohle et al. ${ }^{12}$ have identified a selective R132H-IDH1 inhibitor, e.g., AGI-5198, through a highthroughput screen and demonstrated that AGI-5198 selectively attenuated the growth of glioma xenografts harboring IDH1-
R132H, but not IDH1. In addition, Zheng et al. ${ }^{13}$ have screened a focused library and identified two derivatives of 5-benzyl-1hydroxypyridin-2-one as IDH1-R132H and IDH1-R132C specific inhibitors. Moreover, Davis et al. ${ }^{14}$ have recently identified ML309 as a selective chemical inhibitor of IDH1-R132H and found that it competitively inhibits the binding of $\alpha-\mathrm{KG}$ to the mutant IDH1 enzymes, IDH1-R132H and IDH1-R132C. In the present 

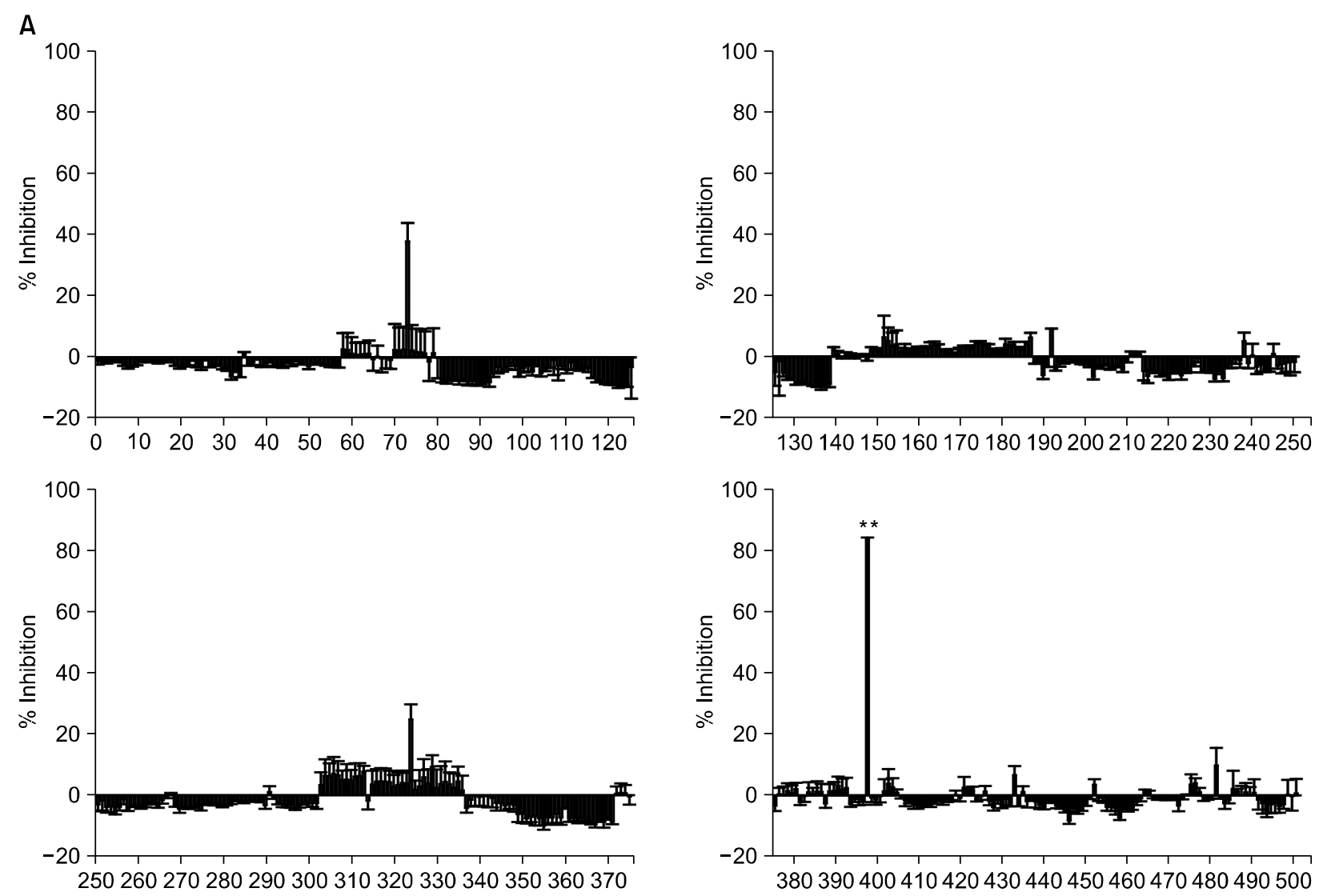

B

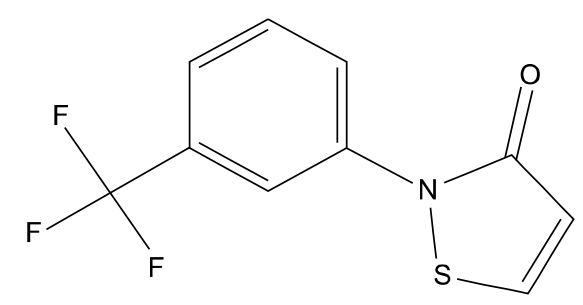

2-(3-trifluoromethylphenyl)isothioazol-3(2H)-one
C

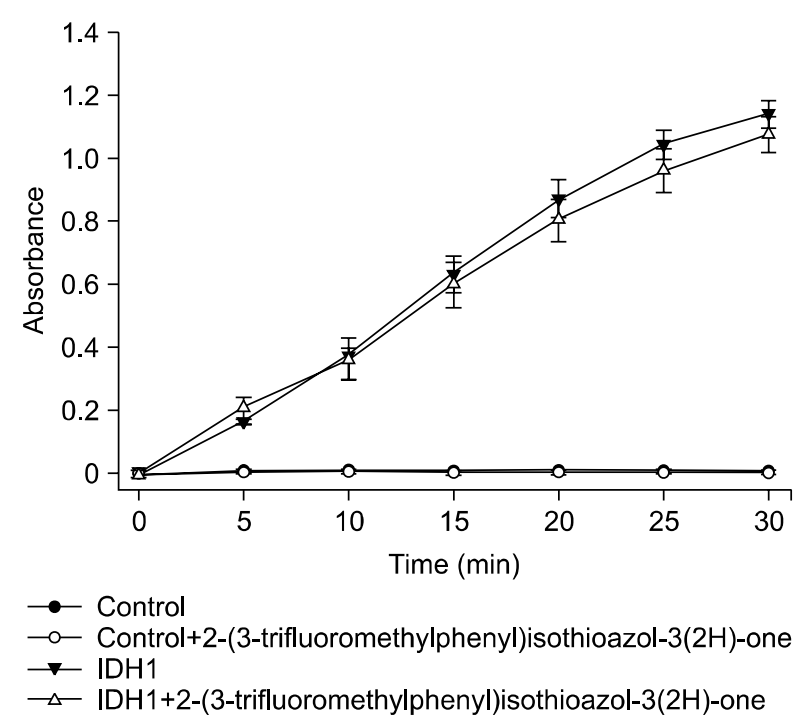

Figure 2. Identification of 2-(3-trifluoromethylphenyl)isothioazol-3(2H)-one as a new selective chemical inhibitor of isocitrate dehydrogenase-1 (IDH1)-R132H. (A) Inhibitory effects of individual compounds derived from a fragment-based chemical library on the IDH1-R132H activity. A newly identified compound (No. 398) bearing the most significant inhibitory effect against IDH1-R132H was marked with a double asterisk. Statistical analysis was conducted by Student t-test and the asterisk indicates a statistical significance with $P<0.01$. (B) Chemical structure of 2-(3-trifluoromethylphenyl)isothioazol-3(2H)-one. (C) 2-(3-trifluoromethylphenyl)isothioazol-3(2H)-one does not interfere with the IDH1 activity. In this experiment, 2-(3-trifluoromethylphenyl) isothioazol-3(2H)-one was added at the concentration of $10 \mu \mathrm{M}$. Control indicates the experiment setup without an addition of purified recombinant IDH1. 
study, we also provide a new selective chemical inhibitor of IDH1-R132H, e.g., 2-(3-trifluoromethylphenyl)isothioazol-3(2H)-one (Fig. 2B), whose core chemical structure is different from the mutant IDH1 selective inhibitors mentioned above. At present, how 2-(3-trifluoromethylphenyl)isothioazol-3(2H)-one selectively inhibits IDH1-R132H is currently unknown and further studies are currently undergoing to address this issue.

\section{ACKNOWLEDGEMENTS}

This work was supported by the GRRC program of Gyeonggi province ([GRRC-DONGGUK2014-B02], Development of novel recombinant enzymes and diagnostic platform).

\section{CONFLICTS OF INTEREST}

No potential conflicts of interest were disclosed.

\section{REFERENCES}

1. Vogelstein B, Papadopoulos N, Velculescu VE, Zhou S, Diaz LA Jr, Kinzler KW. Cancer genome landscapes. Science 2013;339:1546-58.

2. Warburg O. On the origin of cancer cells. Science 1956;123: 309-14.

3. Kloosterhof NK, Bralten LB, Dubbink HJ, French PJ, van den Bent MJ. Isocitrate dehydrogenase-1 mutations: a fundamentally new understanding of diffuse glioma? Lancet Oncol 2011;12:83-91.

4. Yan H, Parsons DW, Jin G, McLendon R, Rasheed BA, Yuan W, et al. IDH1 and IDH2 mutations in gliomas. N Engl J Med 2009;
360:765-73.

5. Xu X, Zhao J, Xu Z, Peng B, Huang Q, Arnold E, et al. Structures of human cytosolic NADP-dependent isocitrate dehydrogenase reveal a novel self-regulatory mechanism of activity. J Biol Chem 2004:279:33946-57.

6. Dang L, Jin S, Su SM. IDH mutations in glioma and acute myeloid leukemia. Trends Mol Med 2010;16:387-97.

7. Zhao S, Lin Y, Xu W, Jiang W, Zha Z, Wang P, et al. Glioma-derived mutations in IDH1 dominantly inhibit IDH1 catalytic activity and induce HIF-1alpha. Science 2009:324:261-5.

8. Dang L, White DW, Gross S, Bennett BD, Bittinger MA, Driggers EM, et al. Cancer-associated IDH1 mutations produce 2-hydroxyglutarate. Nature 2009;462:739-44.

9. Losman JA, Looper RE, Koivunen P, Lee S, Schneider RK, McMahon C, et al. (R)-2-hydroxyglutarate is sufficient to promote leukemogenesis and its effects are reversible. Science 2013;339: 1621-5.

10. Choi C, Ganji SK, DeBerardinis RJ, Hatanpaa KJ, Rakheja D, Kovacs $Z$, et al. 2-hydroxyglutarate detection by magnetic resonance spectroscopy in IDH-mutated patients with gliomas. Nat Med 2012;18:624-9.

11. Venneti S, Thompson CB. Metabolic modulation of epigenetics in gliomas. Brain Pathol 2013;23:217-21.

12. Rohle D, Popovici-Muller J, Palaskas N, Turcan S, Grommes C, Campos C, et al. An inhibitor of mutant IDH1 delays growth and promotes differentiation of glioma cells. Science 2013;340:626-30.

13. Zheng B, Yao Y, Liu Z, Deng L, Anglin JL, Jiang H, et al. Crystallographic investigation and selective inhibition of mutant isocitrate dehydrogenase. ACS Med Chem Lett 2013;4:542-6.

14. Davis MI, Gross S, Shen M, Straley KS, Pragani R, Lea WA, et al. Biochemical, cellular, and biophysical characterization of a potent inhibitor of mutant isocitrate dehydrogenase IDH1. J Biol Chem 2014:289:13717-25. 